\title{
Omega-3 supplementation from pregnancy to postpartum to prevent depressive symptoms: a randomized placebo- controlled trial
}

Juliana dos Santos Vaz ${ }^{1 *}$, Dayana Rodrigues Farias², Amanda Rodrigues Amorim Adegboye ${ }^{3}$, Antonio Egidio Nardi ${ }^{4}$ and Gilberto Kac ${ }^{2}$

\begin{abstract}
Background: Low n-3 polyunsaturated fatty acids (PUFAs) has been linked to depression, but the preventive effect of n-3PUFAs supplementation on maternal depression needs further investigation. We aimed to evaluate the efficacy of a daily dose of n-3 PUFAs supplementation (fish oil) on the prevention of postpartum depression (PPD).

Methods: A randomized, placebo-controlled, double blind trial was designed and nested into a cohort study conducted in Rio de Janeiro, Brazil. Sixty pregnant women identified as being at risk for PPD were invited and randomly assigned to receive fish oil capsules $[1.8 \mathrm{~g}$ (1.08 g of Eicosapentaenoic (EPA) and $0.72 \mathrm{~g}$ of

Docosapentaenoic (DHA) acids)] or placebo (control). The Edinburgh Postnatal Depression Scale (EPDS) was scored at 5-13 (T0, baseline), 22-24 (T1), 30-32 weeks of gestation (T2) and 4-6 weeks' postpartum (T3). Supplementation started at week 22-24 of gestation (T1) and lasted for 16 weeks. Serum fatty acids were assayed to evaluate compliance. Prevalence of EPDS $\geq 11$ was the primary outcome, and mean and changes in EPDS score, length of gestation, and birth weight the secondary outcomes. Linear mixed-effect (LME) and random-intercept logistic regression models were performed to test the effect of fish oil supplementation on prevalence of EPDS $\geq 11$ and EPDS scores variation.

Results: In intention-to-treat (ITT) analysis, at 30-32 weeks' gestation women in the fish oil presented higher serum concentration of EPA, DHA and lower n-6/n-3 ratio comparing to the control group. There were no differences between intervention and control groups in the prevalence of EPDS $\geq 11$, EPDS scores over time, or in changes in EPDS scores from pregnancy to postpartum in either the ITT or per-protocol analyses. Women in the fish oil group with previous history of depression presented a higher reduction on the EPDS score from the second to the third trimester in the fish oil comparing to the control group in the ITT analyses $[-1.0(-3.0-0.0)$ vs. $-0.0(-1.0-3.0), P=0.038)$. These results were confirmed on the LME model ( $\beta=-3.441 ; 95 \% \mathrm{Cl}:-6.532--0.350, P=0.029)$.
\end{abstract}

Conclusion: Daily supplementation of $1.8 \mathrm{~g}$ of $\mathrm{n}-3$ PUFAs during 16 weeks did not prevent maternal depressive symptoms in a sample of Brazilian women.

Trial registration: ClinicalTrials.gov Identifier: NCT01660165. Retrospectively registered on 24 May 2012.

Keywords: Depression; pregnancy, Fatty acids, Omega-3, Randomized controlled trial

\footnotetext{
* Correspondence: juliana.vaz@gmail.com

${ }^{1}$ Faculty of Nutrition, Pelotas Federal University, Rua Gomes Carneiro 1 -

Campus Porto, Pelotas, RS 96160-000, Brazil

Full list of author information is available at the end of the article
} 


\section{Background}

The increase number of clinical and epidemiological studies showing the association between low intake of fish and seafood and increased risk of depression and mental disorders suggests that $\mathrm{n}-3$ polyunsaturated fatty acids (PUFAs) might be a relevant element to treat maternal depression $[1,2]$. N-3 PUFAs are essential components to human diet which must be obtained from dietary sources, as mammals are not able to synthetize de novo [3]. In addition, there is an extensive competition between n- 3 and n- 6 fatty acids for endogenous enzymes that limits the conversion of the $n-3$ precursor alpha-linoleic acid (ALA; 18:3 n-3) into neuro-active of long chain forms, specially eicosapentaenoic (EPA; 20:5 $n-3)$ and docosahexaenoic (DHA; 22:6 n-3) $[4,5]$.

Pregnancy is a period of additional demands of $n-3$ long chain fatty acids due to high maternal transference of EPA (20:5n-3) and DHA (22:6n-3) to the fetus for brain growth and subsequent cognitive development [6]. Selective transportation of these fatty acids across the placenta not only biomagnifies concentrations to the fetus but may also lead to maternal depletion when dietary intake is insufficient $[7,8]$, which can be considered a risk factor for depression onset $[9,10]$.

Maternal depression is recognized as a worldwide public health issue [11]. In high-income countries, 10-20\% women experience depression either during pregnancy or during the first year after birth [12-15]. In low- and lower-middle-income countries the prevalence of depression is higher and ranges from $13 \%$ to $30 \%$ in pregnancy and $13.8 \%$ to $32.9 \%$ in the postnatal period [16]. In Brazil the prevalence of depressive symptoms in the postpartum period among women living in metropolitan areas varies from $20.7 \%$ to $39.4 \%$ [17, 18$]$.

Recent epidemiological studies conducted among Brazilian pregnant women have shown that maternal depression is associated with an unbalanced dietary intake of n-6 and n-3 fatty acids [19] and higher levels of n- 6 serum concentrations [20]. Furthermore, lower serum levels of n3 PUFAs at early pregnancy are associated with reduced fish consumption and lower inter-partum interval [21] and more anxiety symptoms [22]. A number of studies have investigated the effect of fish oil supplementation and depressive symptoms, with conflicting results [23-25]. Findings have not been consistent, neither with respect to major depression in the general populations [26] nor in pregnant and postpartum women [23-25]. Systematic reviews of these interventions also showed mixed results [26-30]. These systematic reviews were challenged by the differences regarding dose and duration of the intervention, type of population, and use of different scales to measure depression across studies, which made it difficult to estimate a pooled effect size from these studies. Therefore, the investigation of the effect of $n-3$ on postpartum depression in further clinical trials, designed in such way that allows comparison with previous trials, is still needed.

The use of fish oil as an alternative treatment for maternal mood disorders deserves special consideration due to potential lack of side effects for fetal development during pregnancy and breastfeeding in addition to potential neurodevelopment benefits [31]. Furthermore, limited access to health care service in low- and lowermiddle-income countries makes urge to identify alternative forms of prevention and treatment for this condition, such as omega-3 supplementation [11]. Thus, this study aimed to evaluate the efficacy of a daily dose of n-3 PUFAs supplementation (fish oil) starting at second trimester of pregnancy and lasting for 16 weeks on the prevention of postpartum depression (PPD) using a randomized, placebocontrolled, double-blind design. We hypothesized that early supplementation of DHA and EPA acts as a protection against $\mathrm{n}-3$ deficiency and may prevent PPD symptoms in women at high-risk.

\section{Methods \\ Participants and setting}

Study participants were recruited from a prospective observational cohort of pregnant women conducted at a public health care center in the city of Rio de Janeiro, Brazil. Enrollment was open from November 2009 to October 2011 and the last follow-up visit occurred in July 2012. The cohort eligibility criteria were defined as follows: (i) being between 5 and 13 weeks of gestation at the time of enrolment; (ii) aged between 20 and 40 years; (iii) be free from any known chronic diseases (other than obesity) such as hypertension and diabetes; (iv) residing in the study catchment area; and (v) intending to continue prenatal care in the public health center.

At the first prenatal visit, a researcher invited women to participate in the cohort study. Ninety percent of eligible women agreed to participate and 299 women were enrolled in the cohort. The cohort study included four waves: at first (T0, baseline $=5-13$ weeks), second $(\mathrm{T} 1=22-24$ weeks $)$ and third trimesters $(\mathrm{T} 2=30$ 32 weeks), and postpartum ( $\mathrm{T} 3=4-6$ weeks' postpartum). Baseline visit consisted of blood collection followed by a psychiatric interview using the Mini International Neuropsychiatric Interview (MINI; version 5.0.0) [32], general questionnaire (socioeconomic data, obstetric history, lifestyle), mental health scales (including the Edinburgh Postnatal Depression Scale - EPDS), dietary intake (food frequency questionnaire, FFQ), and anthropometric assessment (weight and height). All interviews were conducted on the same day of the routine prenatal appointment at the health care center. More details on data collection for the cohort are published elsewhere [33]. 


\section{Trial inclusion and exclusion criteria}

After cohort baseline data collection those women who reported a past history of depression or presented a depressive symptom score at baseline $\geq 9$ based on the EPDS were considered as being at risk for PPD and potential candidates for the randomized clinical trial (RCT). Women that were depressed or presented psychotic symptoms, or had past history of mania or hipomania, were at suicidal risk, taking any psychiatric medication (as anti-depressive and anxiolytic) or being seen by a psychologist or psychiatrist were excluded. Furthermore, we also considered the exclusion of women taking any oil supplementation (as fish oil, flaxseed oil or cod liver oil) and those with fish or seafood allergy. However, no such cases were identified.

\section{Trial design and randomization}

The design comprised a RCT nested to the observational cohort. Eligible women identified after the cohort baseline were randomly assigned to receive either fish oil or placebo capsules. The supplementation started after the second follow-up visit (T1) and lasted for 16 weeks. The randomization was performed by a researcher not involved in the data collection using the participant identification (subject ID) after stratification for EPDS score and previous history of depression. Participants and all research assistants and technicians responsible for running both the cohort study and the RCT were blinded to the study allocation.

\section{Supplementation and compliance}

Participants in the RCT received 6 gelatin capsules (1 $\mathrm{g}$ each) per day containing either fish oil (intervention) or soybean oil (control) for 16 consecutive weeks. The capsules were identical regarding color, shape, size and packing. The fish oil capsules contained a total dose of $1.8 \mathrm{~g}$ of $\mathrm{n}-3$ per day (1.08 $\mathrm{g}$ of EPA and $0.72 \mathrm{~g}$ of DHA). Both capsules were deodorized, and contained $0.2 \mathrm{mg} / \mathrm{g}$ of vitamin $\mathrm{E}$ as antioxidant. The fish oil supplement and its placebo were manufactured by Galena Nutrition ${ }^{\circledR}$ Química Industrial, São Paulo, Brazil.

Women received individual packages containing the daily dose of supplementation and were advised to take three capsules at lunch and three capsules at dinner. Within the first week, a research assistant contacted participants from both groups by phone to verify compliance and possible side effects using a standardized questionnaire. Supply of capsules was provided at week2 , week-8 (3rd trimester of follow-up visit), and week-12. Week-2 and week-12 appointments were schedule solely to provide batch of supplements and assess compliance. All participants consumed $400 \mu \mathrm{g} / \mathrm{d}$ of folic acid from the beginning of pregnancy, and $60 \mathrm{mg} / \mathrm{d}$ ferrous sulphate from the 2nd trimester until delivery, as provided during standard prenatal care in Brazil. Participants were asked to not alter their usual dietary habits and not consume any supplements other then the ones provided by the research team and prenatal care service.

To assess compliance, women were advised to return all empty supplement packages every visit, and a research assistant estimated the number of capsules women had taken during the interval. Additionally, serum fatty acid composition analysis was assayed as a biological marker of compliance.

\section{Depressive symptom measurements}

The first cohort follow-up (T1) was considered baseline to the RCT. At this time point, the EPDS scale was administered and repeated after 8 weeks (at third trimester visit T2) and 4-6 weeks' postpartum (T3 - end-point). This scale has been previously validated for use in pregnancy [34]. Despite EPDS being considered as a screening tool rather than a diagnostic instrument for depression, this scale is widely used and it enables comparison with previous trials [23-25, 35]. The Portuguese version of the EPDS was validated in a sample of mothers from Pelotas, southern Brazil [36]. According to the validation study, the cutoff of $\geq 9$ presents $91.3 \%$ of sensitivity and $54.7 \%$ of specificity for detecting depression compared to a semistructured interview based on the International Statistical Classification of Disease and Related Health Problems (10th Revision) administered by mental health care professionals (gold standard) [37]. At the study end-point, for screening of moderate and severe cases of PPD, the cutoff point of $\geq 11$ ( $83.8 \%$ of sensitivity and $74.7 \%$ of specificity) [36] was used.

Psychiatric interviews were conducted at T0 using the MINI [32]. This instrument consist of a standard model of a brief (15-30 min) structured interview for the evaluation of the existence of Axis I psychiatric disorders according to the Diagnostic and Statistical Manual of Mental Disorders [38]. The interview is divided into 16 modules (A-P), each one containing questions that represent different psychiatric disorders. The patients must answer 'yes' or 'no' to each of the questions; at the end of each module, and the diagnostic (yes/no) is provided. The interviews were performed by physicians and postgraduate students trained for this purpose.

\section{Blood sample}

Fasting blood samples $(5 \mathrm{~mL})$ were collected by a trained technician throughout gestation (T0, T1 and T2). Serum concentrations of EPA and DHA were used to assess compliance to the fish oil supplementation, as both fatty acids measurements are used as biological markers for dietary intake [39]. The serum fatty acids composition was determined using a high-throughput robotic direct methylation method coupled with fast gas-liquid chromatography. The method was developed and validated 
by the Section of Nutritional Neurosciences, Laboratory of Membrane Biochemistry and Biophysics of the National Institute of Health (NIH, Bethesda, MD, USA), where the samples were analyzed $[40,41]$.

\section{Covariates}

A standardized and pre-piloted questionnaire was applied at the cohort baseline to collect the following information: age (years), schooling (years), marital status (married or stable partnership/single), smoking habit (no/yes), history of depression (yes/no), alcohol consumption (no/yes), monthly per-capita family income, and parity (number of parturition).

The early pre-pregnancy body mass index (BMI, $\mathrm{kg} / \mathrm{m}^{2}$ ) was determined from self-reported pre-pregnancy weight $(\mathrm{kg})$, and height $(\mathrm{m})$ measured at baseline (Seca Ltd., Hamburg, Germany). Anthropometric measurements were collected by trained interviewers according to standardized procedures [42].

A validated semi-quantitative FFQ consisting of 81 food items was administered at baseline (at 1st trimester - referring to prior 6 months of habitual diet) and at the third trimester of pregnancy (referring to dietary habits from second to third trimester) [43, 44]. At baseline, predominant dietary sources of long-chain n-3 PUFAs identified were fresh fish, canned tuna/sardines, eggs and red meat [45].

Gestational age was initially determined based on the reported date of the last menstrual period, and lately confirmed with the first ultrasound performed before 24 weeks of gestation. Obstetric outcomes such as length of gestation and birth weight were extracted from obstetric records.

\section{Sample size}

The original sample size calculation was based on a prevalence of postpartum depression ranging from 26.9 to $39.9 \%$ according the previous studies conducted in similar population $[17,46]$. We designed this study to detect a $5 \%$ reduction in the prevalence of EPDS $\geq 11$ from baseline of $27 \%$ to $22 \%$ after the study intervention, compared with controls. The required sample size ranged from 22 to 43 for each treatment group (fish oil and control). Assuming an attrition rate of $15 \%$ due to dropouts and gestational complications, 25 to 51 participants were needed in each treatment group.

\section{Statistical analyses}

The prevalence of depressive symptoms (EPDS score $\geq 11$ ) was the primary outcome, while the mean and absolute changes in EPDS score and length of gestation, and birth weight were the secondary outcomes.

The data distribution was analyzed according to the Shapiro-Wilk test. Socio-economic, demographic, nutritional and gestational outcome characteristics of women in both fish oil and control groups were described using means and standard deviations or medians and interquartile ranges for symmetric and asymmetric variables, respectively. Normal distributed variables were analyzed using Student t-test or qui-square test and asymmetric variables were analyzed using Mann-Whitney $\mathrm{U}$ test.

We compared the serum fatty acid composition between fish oil and control groups at T0 and T1, and tested the compliance with fish oil supplementation at T2. Comparisons were performed using Mann Whitney $U$ test.

The prevalence of depressive symptoms and mean score of EPDS were compared at T0, T1, T2 and T3 between fish oil and control groups using Mann-Whitney U test, Fisher exact test or chi-square. The absolute change $(\Delta)$ in EPDS score between end-point (T3) and T0 (cohort baseline) and between end-point (T3) and T1 (trial baseline) were tested using Mann-Whitney U test.

We performed two different longitudinal regression procedures. First, a random-intercept and slope logistic regression model having longitudinal changes in depressive symptoms (EPDS score $\geq 11 /<11$ ) as the outcome and the intervention groups as the main exposure was performed. Secondly, linear mixed-effect (LME) regression models were used to evaluate the effect of fish oil supplementation on EPDS continuous score change during pregnancy and postpartum. In the LME models, the time (weeks elapsed after conception) was included in all the models as random effects to adjust for the overall and individual variations in the EPDS score over time.

The longitudinal regression model 1 included interactions terms between groups of intervention (fish oil vs. placebo) and time [random-intercept logistic regression $=2$ nd trimester (reference category) vs. 3rd trimester or postpartum; LME = continuous weeks elapsed after conception] in order to evaluate if the EPDS scores (continuous or categorical) change over time were different depending on the supplement group. Results from model 2 were further adjusted for the following confounders: parity, education, skin color and early prepregnancy BMI.

All analyses were performed using both the pre-protocol and intention-to-treat (ITT) procedures. The ITT population included all women randomized, regardless of whether or not they dropped out or fully adhered to the intervention, while the per-protocol population included all women who completed the trial at each time point.

The analyses described above were repeated to compare results considering a subgroup composed of women with previous history of depression.

Statistical analyses were performed using STATA version 12.0 (Stata Corp., College Station, Texas, USA). Values were considered statistically significant when the $p$-value was lower than 0.05 . 


\section{Results}

\section{Flowchart of participants}

Sixty women were randomized to receive the intervention after the second trimester data collection was performed. After randomization (second trimester visit), there was a random loss of 6 women in the fish oil ( 2 transferred pre-natal care to another health center, 1 reported stillbirth, 3 exceeded thirteen weeks of gestation after ultrasound was performed) and 4 in the placebo group (1 transferred pre-natal care assistant to another health center, 1 reported miscarriage and 2 missed 2nd trimester visit,) for reasons apparently not related to the study. In addition, 1 woman in the fish oil group declined participation in the RCT. Even though randomized, all these cases did not receive the intervention. After the trial commencement, 12 women dropped out the intervention (7 women from fish oil and 5 from control group), 1 woman in each intervention group was excluded (fish oil group = lost her capsules; control group = reported preeclampsia) and 1 woman in the control group had a stillbirth. At the postpartum visit, 2 women in the fish oil group missed the appointment. A total of 32 women completed the trial (Fig. 1).

\section{Baseline characteristics and compliance}

At the baseline, there were no differences between groups, with the exception of skin color. Non-white women were more frequent in the control group (82.1\%) compared with the fish oil (59.4\%) (Table 1).

Overall, capsules were well accepted by pregnant women. Eight women ( 4 at the fish oil and 4 at the control group) reported nausea or vomiting, and 1 reported difficulty to swallowing the capsules. No other adverse effect was reported. Compliance was not different between groups among women who have received the intervention, with an average of $74.4 \pm 24.5 \%$ and $73.5 \pm 20.9 \%$ of capsules offered being consumed by the supplemented and control group, respectively ( $p=0.903 ; t=0.123)$. We also did not find statistically significant differences in compliance between fish oil and control groups when only women who have completed the trial were considered $(82.4 \pm 15.6 \%$ vs. $79.1 \pm 16.9 \% ; p=0.559, t=0.509$, respectively) (Results not shown).

After eight weeks of supplementation (T2) women in the fish oil group presented higher serum concentration of total n-3, EPA, DHA and lower n-6/n-3 ratio comparing to the control group (Table 2).

\section{Depressive symptoms and secondary outcomes}

The prevalence of EPDS $\geq 11$ did not differ significantly between fish oil and control at any time in pregnancy or postpartum for neither the ITT or per protocol analysis, nor even when analysis considered only those with previous history of depression (Table 3).
There were no differences between fish oil and control group in the EPDS scores over time, as well as change in EPDS score from pregnancy to postpartum for neither the ITT or per protocol analysis. It was observed a higher reduction on the EPDS score from the second to the third trimester in the fish oil comparing to the control group, in the ITT analyses $[-1.0(-3.0-0.0)$ vs. -0.0 (-1.0-3.0), $P=0.038$ ) (Table 4).

A sharper decline was observed in EPDS score from the second trimester to postpartum for women who reported previous history of depression before pregnancy in the fish oil when compared to the control group ( $ß=-2.665$, 95\% CI: $-4.774--0.556)$ (Fig. 2).

No effect of the intervention on EPDS $\geq 11$ was found, neither on the ITT or per-protocol analysis, or when the analyses were performed for those with previous history of depression (Table 5).

No effect of the intervention on EPDS scores was found between T1 and T2 and T1 and T3 for ITT when intervention and the control group were compared. Similar results were observed for the per-protocol analysis. We found a significant reduction from T1 to T3 in the ITT analysis when only women with previous history of depression were considered $(\beta=-3.441 ; 95 \% \mathrm{CI}$ : $-6.532--0.350, P=0.029$ ) (Table 6).

No differences in gestational length $(39.0 \pm 1.9$ vs. $39.1 \pm 1.8, P=0.859)$ and birth weight $(3322.7 \pm 561.6$ vs. $3210 \pm 553.1, P=0.456)$ were observed between fish oil and control groups (Results not shown).

\section{Discussion}

Our findings showed that in a sample of pregnant women at risk for PPD and low fish intake a daily supplementation of $1.8 \mathrm{~g}$ of $\mathrm{n}-3$ per day (1.08 $\mathrm{g}$ of EPA and $0.72 \mathrm{~g}$ of DHA) had no significant effect on mean depression scores and occurrence of major depressive symptoms during pregnancy and early postpartum. Despite significant increase in serum levels of EPA, DHA and lower $n-6 / n 3$ ratio in the third trimester these changes did not affect EPDS scores at early postpartum. These findings are in line with some earlier preventive trials $[23,35,47,48]$. In contrast, we found that women in the fish oil group with previous history of depression presented a sharper decline in the EPDS score from the second trimester to postpartum comparing to the control group. These results were confirmed by the multiple longitudinal regression model.

One previous RCT with 138 women investigating whether $200 \mathrm{mg}$ DHA/day supplementation in the first four months after delivery could prevent postpartum depression found no significant results [47]. One potential explanation might be the late initiation of the supplementation, as compared with the gradual decline of maternal DHA status during pregnancy. However, another 


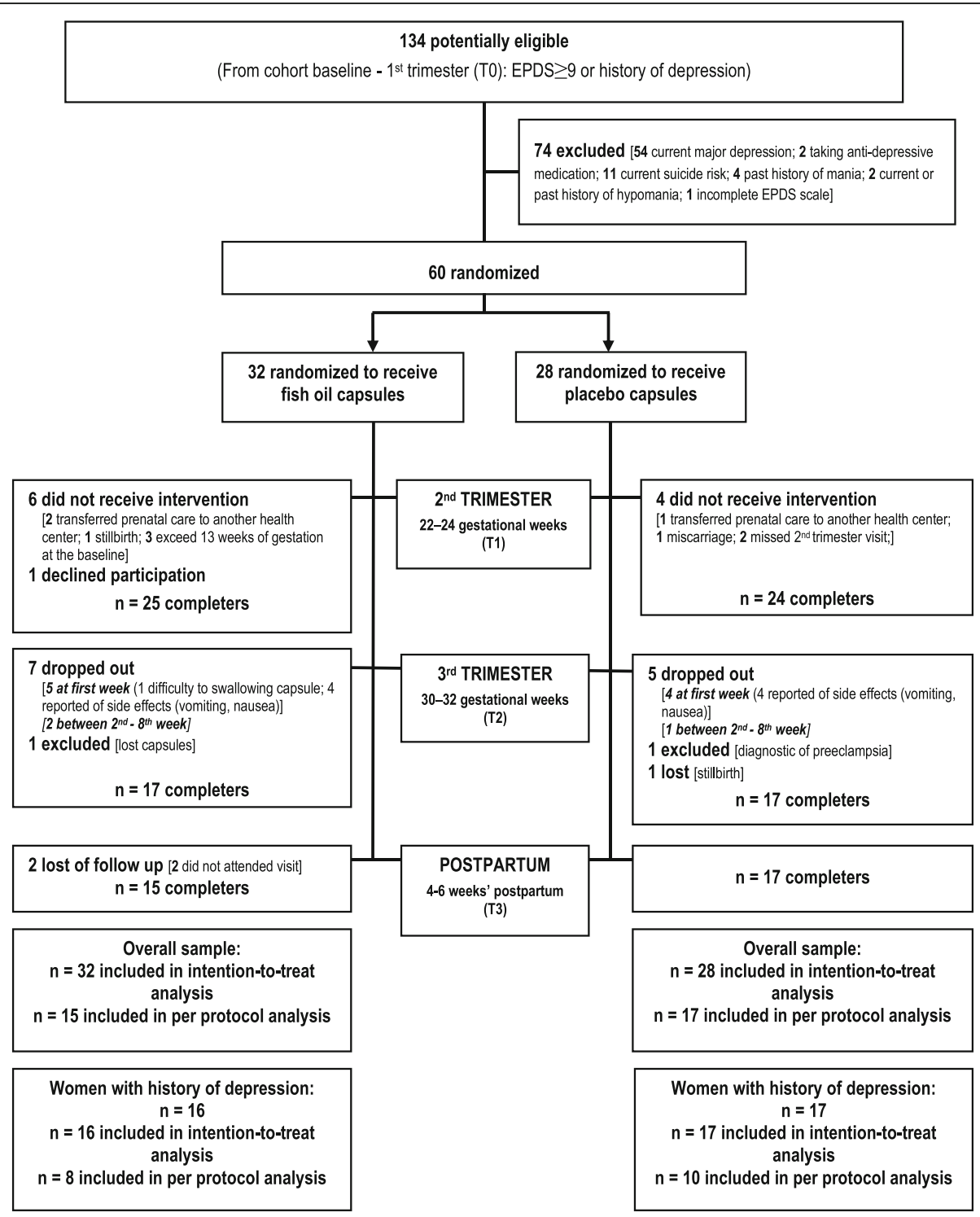

Fig. 1 Flow of participant through the randomized clinical trial

RCT offering $220 \mathrm{mg}$ DHA/day $(n=42)$ or DHA and arachidonic acid (20:4n-6; $n=41)$ (both $220 \mathrm{mg} /$ day) from week 16 of gestation up to 12 weeks postpartum did not find significant results related to prevention of postpartum depressive symptoms either [35].

It has also been argued that EPA has a greater effect in preventing depression and alleviating symptoms than DHA [49]. However, a three arm trial $(n=42$ in each arm) offering daily supplementation of 1) EPA-rich fish oil capsules (4:1 ratio EPA/DHA, $1060 \mathrm{mg}$ EPA plus 274 mg DHA); 2) DHA-rich oil capsules (4:1 ratio DHA/EPA, $900 \mathrm{mg}$ DHA plus $180 \mathrm{mg}$ EPA), and 3) placebo from 12 to 20 weeks of gestation to 8 weeks postpartum found that neither EPA nor DHA supplementation prevented depressive symptoms [48].
It is worth discussing the placebo effect, which all RCTs are subject to. In the case of mood disorders, depressive symptoms may be masked by simply participating in a trial with increased contact with researchers or health care professionals, which creates a therapeutic environment that activates placebo benefits in both trial groups and possibly resulting in spontaneous remission of depressive symptoms [50].

Most of the observational studies that investigated depression from pregnancy to postpartum period reported a significant decrease in depressive scores over time $[12,51]$. This finding is in line with those results observed in our main cohort study [33, 52, 53]. Some authors suggested that a decrease in depression may be due to family and social support and coping behavior and skills 
Table 1 Socio-economic, demographic and nutritional characteristics between fish oil (EPA and DHA) and control groups at the cohort baseline study (TO) (Intention-to-treat analysis)

\begin{tabular}{|c|c|c|c|}
\hline Variable & Fish oil Supplement $(n=32)$ & Control $(n=28)$ & $p$-value \\
\hline Age (years) ${ }^{a}$ & $25.5(22.0-34.5)$ & $27.0(21.0-31.0)$ & 0.795 \\
\hline Gestational age (weeks) ${ }^{b}$ & $9.7(2.3)$ & $9.1(2.5)$ & 0.336 \\
\hline Education (years) ${ }^{a}$ & $11.0(7.0-11)$ & $8.0(7.5-10.5)$ & 0.070 \\
\hline Family income (US \$) ${ }^{\text {a }}$ & $263.2(181.9-383.0)$ & $304.1(180.7-379.8)$ & 0.811 \\
\hline \multicolumn{4}{|l|}{ Skin color ${ }^{c}$} \\
\hline White & $13.0(40.6)$ & $5.0(17.9)$ & \\
\hline Non-white & $19.0(59.4)$ & $23.0(82.1)$ & 0.055 \\
\hline \multicolumn{4}{|l|}{ Parity (number of parturition) ${ }^{c}$} \\
\hline $0-1$ & $26.0(81.2)$ & $18.0(64.3)$ & \\
\hline$\geq 2$ & $6.0(18.8)$ & $10.0(35.7)$ & 0.138 \\
\hline Early pre-pregnancy BMI $\left(\mathrm{kg} / \mathrm{m}^{2}\right)^{a}$ & $23.9(22.6-27.5)$ & $23.1(21.8-27.5)$ & 0.213 \\
\hline Total dietary PUFAs (g/day) ${ }^{a}$ & $11.3(8.0-14.9)$ & $12.1(9.4-17.0)$ & 0.334 \\
\hline Edinburgh Postnatal Depression Scale (Score) ${ }^{a}$ & $10.0(9.0-13.0)$ & $10.5(9.5-13.5)$ & 0.621 \\
\hline \multicolumn{4}{|l|}{ History of depression ${ }^{c}$} \\
\hline No & $16.0(50.0)$ & $11.0(39.3)$ & \\
\hline Yes & $16.0(50.0)$ & $17.0(60.7)$ & 0.405 \\
\hline
\end{tabular}

BMI Body mass index, PUFAs polyunsaturated fatty acids.

${ }^{a}$ Median (interquartile range); $p$-value refers to the Mann-Whitney $\mathrm{U}$ test.

${ }^{\mathrm{b}}$ Mean (standard deviation); $p$-value refers to the Student $t$ test.

${ }^{\mathrm{c}}$ Absolute frequency (\%); $p$-value refers to the qui-square test.

Table 2 Longitudinal variation of median serum fatty acids during pregnancy between fish oil (EPA and DHA) and control groups (T0, T1 and T2). (Intention-to-treat analysis)

\begin{tabular}{|c|c|c|c|}
\hline Fatty acids $(\mu \mathrm{g} / \mathrm{mL})$ & Fish oil Supplement $(n=32)$ & Control $(n=28)$ & $p$-value \\
\hline \multicolumn{4}{|l|}{ Total n-6 } \\
\hline T0 & $1018.3(856.8-1159.9)$ & $939.7(793.2-1047.7)$ & 0.076 \\
\hline T1 & $1274.8(1098.0-1500.6)$ & $1252.9(1109.3-1404.2)$ & 0.563 \\
\hline T2 & $1356.1(1123.3-1551.3)$ & $1362.1(1212.6-1553.9)$ & 0.678 \\
\hline \multicolumn{4}{|l|}{ Total n-3 } \\
\hline T0 & $94.7(78.4-110.6)$ & $95.1(83.1-104.9)$ & 0.976 \\
\hline T1 & $112.6(95.0-127.3)$ & $116.2(105.0-132.2)$ & 0.524 \\
\hline $\mathrm{T} 2$ & $154.7(109.6-194.2)$ & $121.1(94.5-131.9)$ & 0.020 \\
\hline \multicolumn{4}{|l|}{$n-6 / n-3$ ratio } \\
\hline T0 & $11.4(10.3-12.2)$ & $10.3(8.9-11.7)$ & 0.108 \\
\hline T1 & $11.7(9.9-13.0)$ & $10.3(9.7-12.4)$ & 0.328 \\
\hline $\mathrm{T} 2$ & $9.5(7.0-11.9)$ & $11.6(10.0-12.6)$ & 0.004 \\
\hline \multicolumn{4}{|l|}{ Eicosapentaenoic acid } \\
\hline T0 & $9.2(7.0-11.2)$ & $9.5(6.5-12.3)$ & 0.903 \\
\hline T1 & $8.7(6.8-10.7)$ & $8.5(6.7-12.8)$ & 0.813 \\
\hline T2 & $11.9(7.6-26.4)$ & $7.5(4.3-11.0)$ & 0.005 \\
\hline \multicolumn{4}{|l|}{ Docosahexaenoic acid } \\
\hline T0 & $56.8(47.1-67.4)$ & $57.4(48.9-68.3)$ & 0.844 \\
\hline T1 & $67.5(61.6-81.0)$ & $75.5(59.8-84.6)$ & 0.505 \\
\hline $\mathrm{T} 2$ & $97.1(66.0-120.0)$ & $73.5(62.8-83.9)$ & 0.021 \\
\hline
\end{tabular}

Evaluation was conducted at: T0 = 5-13 gestational weeks (cohort baseline); T1 = 22-24 gestational weeks (randomized clinical trial baseline); T2 = 30-32 gestational weeks. a $\mathrm{p}$-value refers to Mann Whitney U test; EPA Eicosapentaenoicacid, DHA Docosahexaenoicacid 
Table 3 Depressive symptoms (EPDS score $\geq 11$ ) during pregnancy and postpartum between fish oil (EPA and DHA) and control groups (TO, T1, T2, and T3)

\begin{tabular}{|c|c|c|c|c|c|c|c|c|c|c|c|c|c|}
\hline & \multirow{3}{*}{ N (\%) } & \multicolumn{3}{|l|}{ T0 } & \multicolumn{3}{|l|}{ T1 } & \multicolumn{3}{|l|}{$\mathrm{T} 2$} & \multicolumn{3}{|l|}{ T3 } \\
\hline & & \multicolumn{2}{|c|}{ Depressive symptoms } & \multirow[t]{2}{*}{$p^{a}$} & \multicolumn{2}{|c|}{ Depressive symptoms } & \multirow[t]{2}{*}{$p^{a}$} & \multicolumn{2}{|c|}{ Depressive symptoms } & \multirow[t]{2}{*}{$p^{a}$} & \multicolumn{2}{|c|}{ Depressive symptoms } & \multirow[t]{2}{*}{$p^{a}$} \\
\hline & & $n$ & Prevalence & & $n$ & Prevalence & & $n$ & Prevalence & & $n$ & Prevalence & \\
\hline \multicolumn{14}{|c|}{ Overall sample } \\
\hline \multicolumn{14}{|c|}{ Intention-to-treat } \\
\hline Control & $28(46.7)$ & 14 & 50.0 & & 7 & 25.0 & & 8 & 28.6 & & 7 & 25.0 & \\
\hline Fish oil & $32(53.3)$ & 15 & 46.9 & 0.809 & 12 & 37.5 & 0.299 & 11 & 34.4 & 0.630 & 8 & 25.0 & 1.000 \\
\hline \multicolumn{14}{|c|}{ Per protocol } \\
\hline Control & $17(53.1)$ & 9 & 52.9 & & 4 & 23.5 & & 5 & 29.4 & & 3 & 17.6 & \\
\hline Fish oil & $15(46.9)$ & 9 & 60.0 & 0.688 & 8 & 53.3 & 0.082 & 7 & 46.7 & 0.314 & 5 & 33.3 & 0.306 \\
\hline \multicolumn{14}{|c|}{ Women with previous history of depression } \\
\hline \multicolumn{14}{|c|}{ Intention-to-treat } \\
\hline Control & $17(51.5)$ & 9 & 52.9 & & 5 & 29.4 & & 7 & 41.2 & & 7 & 41.2 & \\
\hline Fish oil & $16(48.5)$ & 6 & 37.5 & 0.373 & 7 & 43.8 & 0.392 & 7 & 43.8 & 0.579 & 5 & 31.3 & 0.554 \\
\hline \multicolumn{14}{|c|}{ Per protocol } \\
\hline Control & $10(55.6)$ & 5 & 50.0 & & 3 & 30.0 & & 4 & 40.0 & & 3 & 30.0 & \\
\hline Fish oil & $8(44.4)$ & 4 & 50.0 & 1.000 & 5 & 62.5 & 0.168 & 4 & 50.0 & 0.671 & 3 & 37.5 & 0.737 \\
\hline
\end{tabular}

Evaluation was conducted at: T0 $=5-13$ gestational weeks (cohort baseline); $\mathrm{T} 1=22-24$ gestational weeks (randomized clinical trial baseline); $\mathrm{T} 2=30-32$ gestational weeks; $\mathrm{T3}=4-6$ weeks' postpartum.

EPDS Edinburgh Postnatal Depression Scale, EPA Eicosapentaenoic acid, DHA Docosahexaenoic acid.

${ }^{a} \mathrm{p}$-value refers to chi-square test or Fisher exact test.

Table 4 Longitudinal variation of EPDS score during pregnancy and postpartum between fish oil (EPA and DHA) and control groups

\begin{tabular}{lllll}
\hline EPDS score & & Absolute change in EPDS score \\
\hline T0 & T1 & T2 & T3 & $\Delta(\mathrm{T3}-\mathrm{T} 1) \quad \Delta(\mathrm{T} 3-\mathrm{T} 0)$
\end{tabular}

Overall sample

Intention-to-treat

\begin{tabular}{|c|c|c|c|c|c|c|}
\hline Control $(n=28)$ & $10.5(9.5-13.5)$ & $8.5(6.0-10.5)$ & $7.0(5.5-11.0)$ & $6.0(5.0-11.0)$ & $-1.0(-3.5-1.5)$ & $-4.0(-8.0-0.0)$ \\
\hline Fish oil $(n=32)$ & $10.0(9.0-13.0)$ & $9.0(5.5-13.0)$ & $6.5(4.0-12.0)$ & $6.5(3.5-10.5)$ & $-1.0(-4.0-0.0)$ & $-4.0(-6.5-0.0)$ \\
\hline p-value ${ }^{1}$ & 0.612 & 0.721 & 0.607 & 0.612 & 0.512 & 0.748 \\
\hline \multicolumn{7}{|l|}{ Per protocol } \\
\hline Control $(n=17)$ & $11.0(9.0-15.0)$ & $8.0(6.0-10.0)$ & $7.0(5.0-11.0)$ & $6.0(5.0-7.0)$ & $-1.0(-4.0-1.0)$ & $-5.0(-8.0--3.0)$ \\
\hline Fish oil $(n=15)$ & $11.0(10.0-14.0)$ & $11.0(5.0-14.0)$ & $10.0(4.0-15.0)$ & $8.0(5.0-12.0)$ & $-1.0(-5.0-1.0)$ & $-4.0(-8.0-0.0)$ \\
\hline p-value ${ }^{1}$ & 0.675 & 0.178 & 0.635 & 0.216 & 0.955 & 0.404 \\
\hline \multicolumn{7}{|c|}{ Women with previous history of depression } \\
\hline \multicolumn{7}{|l|}{ Intention-to-treat } \\
\hline Control $(n=17)$ & $11.0(10.0-15.0)$ & $9.0(5.0-12.0)$ & $7.5(5.5-11.0)$ & $7.0(5.0-14.0)$ & $0.0(-1.0-3.0)$ & $-3.0(-8.0-0.0)$ \\
\hline Fish oil $(n=16)$ & $10.0(7.0-12.5)$ & $8.5(5.5-13.5)$ & $9.0(5.0-15.0)$ & $7.0(3.0-11.0)$ & $-1.0(-3.0-0.0)$ & $-1.0(-6.0-0.0)$ \\
\hline$p$-value ${ }^{1}$ & 0.318 & 0.527 & 0.709 & 0.322 & 0.038 & 0.201 \\
\hline \multicolumn{7}{|l|}{ Per protocol } \\
\hline Control $(n=10)$ & $11.0(8.0-15.0)$ & $7.5(5.0-12.0)$ & $7.5(5.0-11.0)$ & $6.5(5.0-12.0)$ & $-0.5(-2.0-2.0)$ & $-4.5(-8.0-0.0)$ \\
\hline Fish oil $(n=8)$ & $10.5(10.0-15.0)$ & $13.0(6.0-14.5)$ & $12.5(5.0-15.5)$ & $8.5(6.5-12.0)$ & $-1.5(-4.0-0.5)$ & $-4.5(-7.0-1.0)$ \\
\hline p-value ${ }^{1}$ & 0.893 & 0.117 & 0.303 & 0.421 & 0.264 & 0.561 \\
\hline
\end{tabular}

Evaluation was conducted at: T0 $=5-13$ gestational weeks (cohort baseline); $\mathrm{T} 1=22-24$ gestational weeks (randomized clinical trial baseline); $\mathrm{T} 2=30-32$ gestational weeks; T3 = 4-6 weeks' postpartum

Data are presented as median (interquartile range), ${ }^{1} \mathrm{p}$-value refers to Mann Whitney $\mathrm{U}$ test.

EPDS Edinburgh Postnatal Depression Scale, EPA Eicosapentaenoic acid, DHA Docosahexaenoic acid. 


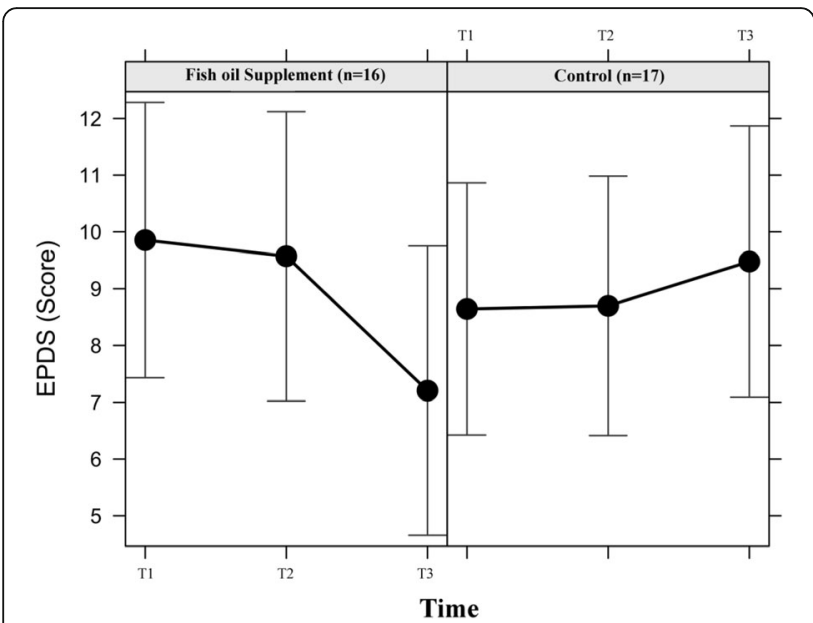

Fig. 2 Longitudinal changes in EDPS score in women with previous history of depression according to intervention group (Intention-to-treat analysis). Note: $\mathrm{T1}=22-24$ gestational weeks (Randomized Clinical Trial baseline); T2 $=30-32$ gestational weeks; $\mathrm{T} 3=4-6$ weeks' postpartum. Data are presented as linear mixed effect coefficient ( $\beta$ ) and 95\% Cl. Pvalue refers to the maximum likelihood estimator. $T 1$ was the reference category. Fish oil supplement group: $\beta_{\text {(12) }}=-0.266 ; 95 \% \mathrm{Cl}=-2.428$ to 1.896; $P=0.809$. $\beta_{(\text {(13) }}=-2.665 ; 95 \% \mathrm{Cl}=-4.774$ to $-0.556 ; P=0.013$.

Control group: $\beta_{(\text {(12) }}=0.046 ; 95 \% \mathrm{Cl}=-2.118$ to $2.210 ; P=0.967$. $\beta_{(\text {(т) }}=0.891 ; 95 \% \mathrm{Cl}=-1.364$ to $3.146 ; P=0.439$

[54] specially observed among second-time mothers [55]. Thus, the possible protective effect of fish oil supplementation could be observed in a time-point later than 46 weeks' postpartum.

The question that hovers over RCTs conducted during pregnancy is the definition of the supplement dose used to treat or prevent mood disorders. Specific recommendations for DHA and EPA supplementation during pregnancy have not been fully established due to insufficient data on the benefits to maternal and infant outcomes (e.g., prematurity). The recommendation of the American Dietetic Association and Dieticians of Canada is that adults, including pregnant and lactating women, should have a combined intake of $500 \mathrm{mg} /$ day of DHA and EPA based on an average intake of $2000 \mathrm{kcal} /$ day [56]. Following the guidance of the American Institute of Medicine [57], seafood intake during pregnancy and lactation should be approximately $200-300 \mathrm{mg}$ DHA/day. The Perinatal Lipid Intake Working Group on behalf of the European Commission has reviewed all available evidence and recommended an intake of $\geq 200 \mathrm{mg} \mathrm{DHA} /$ day for pregnant and lactating women [58]. Since the dietary requirement for DHA and EPA for a healthy pregnancy has not been clearly established, it may be difficult to define the ideal dose for an intervention. Despite fish and seafood products being considered best sources of EPA and DHA, the intake of these food items was low in our population, i.e. $39 \%$ of women in the cohort did not report intake of fresh fish, and 34\% reported intake once a month. Overall, the median dietary intake of EPA and DHA was $0.025 \mathrm{~g}$ (interquartile range $=0.01-0.03$ ) per day and $0.026 \mathrm{~g}$ (interquartile range $=0.0-0.03)$ per day [45] respectively, which was far bellow the recommendations.

Although previous studies have investigated the effect of fish oil supplementation during pregnancy and postpartum on depression [10,23, 24, 35], to the best of our knowledge this is one of the few studies with focus on prevention of postpartum depressive symptoms in women at high risk and provided longer period of supplementation covering the second trimester of pregnancy to early postpartum. The study design (double-blind) reduces the risk of the bias and increases the credibility in the results. An additional strength of this study is the use of biomarkers to evaluate intervention compliance. Our results showed that in the fish oil group serum levels of EPA and DHA significantly were higher and $n-6 / n-3$ ratio was lower in the third trimester compared to the control group. Another important strength of this study is the use of biological samples in the investigation of mood disorders. Serum levels of fatty acids are known as biological markers of dietary intake in epidemiological investigation [39]. The serum composition of essential n-6 and n-3 fatty acids assessed in the first trimester (cohort baseline) partially reflects the usual diet with less interference of the gestational period [45]. A further strength is the use of different instruments, including a validated scale (EPDS) to measure depressive symptoms and a structured interview to assess the diagnosis of major depressive episodes (MINI). Most RCTs investigated depression based solely on scales used for screening of depression [23, 35, 47]. Although EPDS is a self-reported questionnaire, this scale is widely used in psychiatry studies with pregnant women; thus it allows comparisons and enhances the credibility of our findings, as current results were consistent across different scales and outcome measures.

Although the early initiation of the supplementation and relatively high dose of EPA compared to some previous studies, the current RCT had no effect on depressive symptoms during pregnancy and postpartum. This finding might be explained by the fact that the effect is too subtle to be detected even in large sample size RCTs $[23,35,47$, 48]. Although ITT analyses, whereby all patients randomly assigned to one of the study arms are analyzed, regardless of whether or not they completed or received the full intervention, is a rather conservative approach, findings remained unaltered when excluding women who did not receive the intervention or lost to follow up. 
Table 5 Random-intercept longitudinal logistic regression of fish oil (EPA and DHA) supplementation on depressive symptoms (EPDS score $\geq 11$ ) during pregnancy ${ }^{a}$ and postpartum ${ }^{b}$

\begin{tabular}{|c|c|c|c|c|}
\hline & Model $1^{c}$ & $p^{f}$ & Model $2^{\mathrm{d}}$ & $p^{f}$ \\
\hline & $\mathrm{OR}^{\mathrm{e}}(95 \% \mathrm{Cl})$ & & $\beta^{e}(95 \% \mathrm{Cl})$ & \\
\hline Overall sample & & & & \\
\hline Intention-to-treat & & & & \\
\hline Supplement (Control vs. Fish oil) & $3.338(0.347-32.122)$ & 0.297 & $3.376(0.220-51.757)$ & 0.382 \\
\hline Time & & & & \\
\hline T1 vs. T2 & $0.848(0.092-7.776)$ & 0.884 & $1.566(0.195-12.572)$ & 0.673 \\
\hline T1 vs. T3 & $0.119(0.001-10.666)$ & 0.353 & $0.722(0.037-14.110)$ & 0.830 \\
\hline Interaction terms Time \# Supplem & & & & \\
\hline T2 \# Fish oil & $0.375(0.023-6.094)$ & 0.491 & $0.334(0.023-4.871)$ & 0.423 \\
\hline T3 \# Fish oil & $0.112(0.001-8.946)$ & 0.328 & $0.137(0.005-3.824)$ & 0.242 \\
\hline Women with previous history of $\mathrm{c}$ & & & & \\
\hline Intention-to-treat & & & & \\
\hline Supplement (Control vs. Fish oil) & $2.812(0.183-43.201)$ & 0.458 & $2.833(0.223-36.012)$ & 0.422 \\
\hline Time & & & & \\
\hline T1 vs. T2 & $2.358(0.188-29.622)$ & 0.506 & $2.404(0.240-24.095)$ & 0.456 \\
\hline T1 vs. T3 & $1.878(0.066-53.242)$ & 0.712 & $1.848(0.084-40.854)$ & 0.697 \\
\hline Interaction terms Time \# Supplem & & & & \\
\hline T2 \# Fish oil & $0.237(0.007-7.951)$ & 0.422 & $0.239(0.008-7.052)$ & 0.407 \\
\hline T3 \# Fish oil & $0.034(0.0001-7.597)$ & 0.221 & $0.033(0.0002-6.986)$ & 0.213 \\
\hline $\begin{array}{l}\text { Per-protocol analysis had a reduced } \\
\text { EPDS Edinburgh Postnatal Depressio } \\
\text { aPregnancy period comprised T1 ( } 22 \\
\text { bPostpartum period comprised T3 (b } \\
\text { cModel } 1 \text { was adjusted for the intera } \\
{ }^{\mathrm{d}} \text { Model } 2 \text { was additionally adjusted } \mathrm{f} \\
\text { e } \mathrm{f}=\text { Logistic Mixed Effect odds rati } \\
{ }^{\mathrm{f}} \text {-value refers to maximum likelihoo }\end{array}$ & $\begin{array}{l}\text { were not presented du } \\
\text { lence interval, EPA Eicos } \\
\text { weeks - randomized clin } \\
\text { weeks' postpartum); } \\
\text { me (T1 - T3) and fish oi } \\
\text { e, parity, education, ski }\end{array}$ & $\begin{array}{l}\text { esults. } \\
\text { d, } D H A \\
\text { e) and T } \\
\text { y pre-pr }\end{array}$ & $\begin{array}{l}\text { c acid } \\
\text { onal weeks); }\end{array}$ & \\
\hline
\end{tabular}

We found in this Brazilian RCT that women in the fish oil group with previous history of depression presented a significant reduction in the EPDS score from pregnancy to postpartum when results where compared to the control group. Our results are in line with the hypothesis-testing meta-analysis of Hallahan et al. [59] that have shown that the efficacy of omega-3 supplementation has significant beneficial on subjects with a diagnosis of depressive illness compared to placebo, with no benefit observed in non-depressed groups. Depression symptoms are difficult to be measured and changes in mood in non-clinical depressive populations might not necessarily have the same translation as in clinical depressive subjects [59]. This may justify why we only observed a significant difference in EPDS score among those women with previous history of depression, as this condition may represent recurrent cases with periods of normal function between episodes of depression.

Furthermore, higher dosages might be needed, particularly in a population with low EPA and DHA dietary intake. In this study, the maximum commercial available dose in Brazil was used; attempts to obtain capsules with higher
EPA and DHA were made, however national regulations to import pharmaceutical products precluded any possibility to purchase supplements from international industries. To achieve a dose of $1.8 \mathrm{~g}$ of long chain n-3 (DHA + EPA) per day, women needed to take 6 capsules per day. However, compliance was satisfactory despite the fact that 8 women could not tolerate the ingestion of 6 capsules per day due to nausea and vomiting which are common symptoms during pregnancy.

\section{Conclusions}

In a population of Brazilian pregnant women at risk to PPD and low fish intake a daily dose of $1.8 \mathrm{~g}$ of $\mathrm{n}-3$ PUFAs (1.08 g of EPA and $0.72 \mathrm{~g}$ of DHA) for 16 weeks starting at 22-24 weeks of pregnancy did not prevent depressive symptoms in pregnancy and early postpartum. The possible benefit in women with history of depression must be further investigated, as only few trials have particularly focused on high-risk women and results are still conflicting. 
Table 6 Longitudinal effect of fish oil (EPA and DHA) supplementation on EPDS score during pregnancy ${ }^{a}$ and $_{\text {postpartum }}{ }^{b}$

\begin{tabular}{|c|c|c|c|c|}
\hline & Model $1^{c}$ & $p^{f}$ & Model $2^{d}$ & $p^{f}$ \\
\hline & \multicolumn{2}{|l|}{$\beta^{e}(95 \% \mathrm{Cl})$} & \multicolumn{2}{|l|}{$\beta^{\text {e }}(95 \% \mathrm{Cl})$} \\
\hline \multicolumn{5}{|l|}{ Overall sample } \\
\hline \multicolumn{5}{|l|}{ Intention-to-treat } \\
\hline Supplement (Control vs. Fish oil) & $0.597(-1.771-2.965)$ & 0.621 & $0.761(-1.678-3.201)$ & 0.541 \\
\hline \multicolumn{5}{|l|}{ Time } \\
\hline T1 vs. T2 & $-0.480(-2.166-1.157)$ & 0.566 & $-0.481(-2.119-1.157)$ & 0.565 \\
\hline T1 vs. T3 & $-0.894(-2.532-0.743)$ & 0.284 & $-0.890(-2.528-0.748)$ & 0.287 \\
\hline \multicolumn{5}{|l|}{ Interaction terms Time \# Supplement } \\
\hline T2 \# Fish oil & $-0.745(-3.061-1.570)$ & 0.528 & $-0.734(-3.051-1.583)$ & 0.535 \\
\hline T3 \# Fish oil & $-1.647(-3.959-0.665)$ & 0.163 & $-1.651(-3.963-0.662)$ & 0.162 \\
\hline \multicolumn{5}{|l|}{ Per protocol } \\
\hline Supplement (Control vs. Fish oil) & $2.620(-0.642-5.881)$ & 0.115 & $2.446(-0.859-5.752)$ & 0.147 \\
\hline \multicolumn{5}{|l|}{ Time } \\
\hline T1 vs. T2 & $-0.172(-2.368-2.024)$ & 0.877 & $-0.160(-2.294-1.989)$ & 0.884 \\
\hline T1 vs. T3 & $-1.189(-3.435-1.056)$ & 0.299 & $-1.172(-3.470-1.126)$ & 0.318 \\
\hline \multicolumn{5}{|c|}{ Interaction terms - Time \# Supplement } \\
\hline T2 \# Fish oil & $-0.850(-4.058-2.357)$ & 0.603 & $-0.864(-4.003-2.276)$ & 0.590 \\
\hline T3 \# Fish oil & $-1.189(-4.468-2.089)$ & 0.477 & $-1.260(-4.613-2.093)$ & 0.461 \\
\hline \multicolumn{5}{|c|}{ Women with previous history of depression } \\
\hline \multicolumn{5}{|l|}{ Intention-to-treat } \\
\hline Supplement (Control vs. Fish oil) & $1.210(2.033-4.453)$ & 0.465 & $1.175(-2.052-4.401)$ & 0.475 \\
\hline \multicolumn{5}{|l|}{ Time } \\
\hline T1 vs. T2 & $0.054(-2.011-2.120)$ & 0.959 & $0.053(-2.008-2.114)$ & 0.960 \\
\hline T1 vs. T3 & $0.834(-1.242-2.910)$ & 0.431 & $0.843(-1.238-2.924)$ & 0.427 \\
\hline \multicolumn{5}{|c|}{ Interaction terms Time \# Supplement } \\
\hline T2 \# Fish oil & $-0.337(-3.471-2.796)$ & 0.833 & $-0.306(-3.432-2.820)$ & 0.848 \\
\hline T3 \# Fish oil & $-3.484(-6.567--0.401)$ & 0.027 & $-3.441(-6.532--0.350)$ & 0.029 \\
\hline \multicolumn{5}{|l|}{ Per protocol } \\
\hline Supplement (Control vs. Fish oil) & $3.466(-0.497-7.429)$ & 0.087 & $3.623(-0.192-7.438)$ & 0.063 \\
\hline \multicolumn{5}{|l|}{ Time } \\
\hline T1 vs. T2 & $0.099(-2.586-2.785)$ & 0.942 & $0.101(-2.584-2.786)$ & 0.941 \\
\hline T1 vs. T3 & $-0.098(-2.785-2.589)$ & 0.943 & $-0.101(-2.788-2.587)$ & 0.941 \\
\hline \multicolumn{5}{|c|}{ Interaction terms - Time \# Supplement } \\
\hline T2 \# Fish oil & $-0.474(-4.502-3.555)$ & 0.818 & $-0.476(-4.503-3.552)$ & 0.817 \\
\hline T3 \# Fish oil & $-2.398(-6.429-1.632)$ & 0.244 & $-2.400(-6.431-1.631)$ & 0.243 \\
\hline
\end{tabular}

EPDS Edinburgh Postnatal Depression Scale, Cl confidence interval, EPA Eicosapentaenoic acid, DHA Docosahexaenoic acid

aPregnancy period comprised T1 (22-24 gestational weeks) and T2 (30-32 gestational weeks);

'Postpartum period comprised T3 (between 4 and 6 weeks' postpartum);

${ }^{c}$ Model 1 was adjusted for the interaction between time (T1 - T3) and fish oil supplement;

${ }^{\mathrm{d}}$ Model 2 was additionally adjusted for gestational age, parity, education, skin color and early pre-pregnancy BMl;

${ }^{\mathrm{e}} \beta=$ Linear Mixed Effect (LME) coefficient

${ }^{f} \mathrm{p}$-value refers to maximum likelihood estimator. 


\section{Abbreviations}

BMI: Body Mass Index; DHA: Docosapentaenoic acid; EPA: Eicosapentaenoic acid; EPDS: Edinburgh Postnatal Depression Scale; ICD: International Classification of Disease; ID: Identification; ITT: Intention-To-Treat; LME: Linear Mixed-Effect; MINI: Mini International Neuropsychiatric Interview; PPD: Postpartum depression; PUFAs: Polyunsaturated fatty acids; RCT: Randomized Controlled Trial; T: Time

\section{Acknowledgements}

We are extremely grateful to all women who took part in the study, and to all staff at the Heitor Beltrão Health Center, Brazil. We especially thank Capt Joseph Hibbeln, Dr. Yuhong Lin, PhD and James Loewke, BS from the Section of Nutritional Neuroscience, Laboratory of Membrane and Biophysics, $\mathrm{NIH/USA}$ for their laboratory assistance during the fatty acid analyses. GK and AEN are research fellows from the National Council for Scientific and Technological Development (CNPq). JSV and DRF are recipients of scholarship from the Brazilian Coordination for the Training of University Level Personnel (CAPES).

\section{Funding}

The present study was support by Carlos Chagas Filho Foundation for Research Support of Rio de Janeiro State (Grant No. E-26/112.181/2012). The funding institution did not have any role in study design, data collection, analysis, and interpretation of results, writing the manuscript or in the decision to submit the manuscript for publication.

\section{Availability of data and materials}

The dataset supporting the conclusions of this article is not openly available due to confidentiality of information. Consent for publication of raw data was not obtained but fully anonymous dataset can be obtained with corresponding author.

\section{Authors' contributions}

JSV and GK designed the study protocol. AEN was involved in the study design. DRF conducted the statistical analysis, and ARAA provided critical review of the statistical analysis and essential critical revisions of the manuscript. JSV and ARAA wrote the first draft of the manuscript. All authors contributed to and have approved the final manuscript.

\section{Competing interests}

The authors declare that they have no competing interests.

\section{Consent for publication}

Not applicable

\section{Ethics approval and consent to participate}

The Institute of Psychiatry, from the Federal University of Rio de Janeiro (Protocol number: 0012.0.249.000-09), and the Ethics Committee of the Municipal Secretary of Rio de Janeiro city (Protocol number: 0139.0.314.00009) approved the study. All participants signed a two-way term of consent, which was obtained freely and spontaneously, after all necessary clarifications had been provided.

\section{Publisher's Note}

Springer Nature remains neutral with regard to jurisdictional claims in published maps and institutional affiliations.

\footnotetext{
Author details

${ }^{1}$ Faculty of Nutrition, Pelotas Federal University, Rua Gomes Carneiro 1 Campus Porto, Pelotas, RS 96160-000, Brazil. ${ }^{2}$ Institute of Nutrition, Nutritional Epidemiology Observatory, Rio de Janeiro Federal University, Avenida Carlos Chagas Filho, 367, CCS - Bloco J - $2^{\circ}$ andar, sala 29, Cidade Universitária Ilha do Fundão, Rio de Janeiro, RJ 21941-590, Brazil. ${ }^{3}$ Faculty of Science and Technology, University of Westminster, 115 New Cavendish Street, London W1W 6UW, UK. ${ }^{4}$ Laboratory of Panic and Respiration, Institute of Psychiatry, Rio de Janeiro Federal University, Avenida Venceslau Braz, 71 - Botafogo, Rio de Janeiro, RJ 22290-140, Brazil.
}

Received: 8 June 2016 Accepted: 31 May 2017

Published online: 09 June 2017

\section{References}

1. Golding J, Steer C, Emmett P, Davis JM, Hibbeln JR. High levels of depressive symptoms in pregnancy with low omega-3 fatty acid intake from fish. Epidemiology. 2009;20(4):598-603.

2. Vaz Jdos S, Kac G, Emmett P, Davis JM, Golding J, Hibbeln JR. Dietary patterns, n-3 fatty acids intake from seafood and high levels of anxiety symptoms during pregnancy: findings from the Avon longitudinal study of parents and children. PLoS One. 2013;8(7):e67671.

3. Hibbeln JR, Davis JM. Considerations regarding neuropsychiatric nutritional requirements for intakes of omega-3 highly unsaturated fatty acids. Prostaglandins Leukot Essent Fatty Acids. 2009;81(2-3):179-86.

4. Gibson RA, Muhlhausler B, Makrides M. Conversion of linoleic acid and alpha-linolenic acid to long-chain polyunsaturated fatty acids (LCPUFAs), with a focus on pregnancy, lactation and the first 2 years of life. Matern Child Nutr. 2011;7(Suppl 2):17-26.

5. Brenna JT, Salem N Jr, Sinclair AJ, Cunnane SC. Alpha-Linolenic acid supplementation and conversion to $n-3$ long-chain polyunsaturated fatty acids in humans. Prostaglandins Leukot Essent Fatty Acids. 2009; 80(2-3):85-91.

6. Campoy C, Escolano-Margarit MV, Anjos T, Szajewska H, Uauy R. Omega 3 fatty acids on child growth, visual acuity and neurodevelopment. Br J Nutr. 2012;107(Suppl 2):S85-106.

7. Hornstra G. Essential fatty acids in mothers and their neonates. Am J Clin Nutr. 2000;71(5 Suppl):1262S-9S.

8. Koletzko B, Lien E, Agostoni C, Bohles H, Campoy C, Cetin I, et al. The roles of long-chain polyunsaturated fatty acids in pregnancy, lactation and infancy: review of current knowledge and consensus recommendations. J Perinat Med. 2008;36(1):5-14

9. Otto SJ, de Groot RH, Hornstra G. Increased risk of postpartum depressive symptoms is associated with slower normalization after pregnancy of the functional docosahexaenoic acid status. Prostaglandins Leukot Essent Fatty Acids. 2003:69(4):237-43.

10. Rees AM, Austin MP, Owen C, Parker G. Omega-3 deficiency associated with perinatal depression: case control study. Psychiatry Res. 2009;166(2-3):254-9.

11. WHO: World Health Organization. Mental health aspects of women's reproductive health: a global review of the literature. Geneva: WHO Press, World Health Organization; 2009. http://apps.who.int/iris/bitstream/10665/ 43846/1/9789241563567_eng.pdf.

12. Evans J, Heron J, Francomb H, Oke S, Golding J. Cohort study of depressed mood during pregnancy and after childbirth. BMJ. 2001;323(7307):257-60.

13. Marcus SM, Flynn HA, Blow FC, Barry KL. Depressive symptoms among pregnant women screened in obstetrics settings. J Women's Health (Larchmt). 2003:12(4):373-80.

14. Glavin K, Leahy-Warren P. Postnatal depression is a public health nursing issue: perspectives from norway and ireland. Nurs Res Pract. 2013;2013:813409.

15. Shidhaye P, Giri P. Maternal depression: a hidden burden in developing countries. Ann Med Health Sci Res. 2014;4(4):463-5.

16. Fisher J, Cabral de Mello M, Patel V, Rahman A, Tran T, Holton S, et al. Prevalence and determinants of common perinatal mental disorders in women in low- and lower-middle-income countries: a systematic review. Bull World Health Organ. 2012;90(2):139G-49G.

17. Ruschi GEC, Sun SY, Mattar R, Chambo Filho A, Zandonade E, Lima VJ. Aspectos epidemiológicos da depressão pós-parto em amostra brasileira. Rev psiquiatr Rio Gd Sul. 2007;29(3):274-80.

18. Tannous L, Gigante LP, Fuchs SC, Busnello ED. Postnatal depression in southern Brazil: prevalence and its demographic and socioeconomic determinants. BMC Psychiatry. 2008:8:1.

19. da Rocha CM, Kac G. High dietary ratio of omega- 6 to omega-3 polyunsaturated acids during pregnancy and prevalence of post-partum depression. Matern Child Nutr. 2012;8(1):36-48.

20. Vaz JS, Kac G, Nardi AE, Hibbeln JR. Omega- 6 fatty acids and greater likelihood of suicide risk and major depression in early pregnancy. J Affect Disord. 2014;152-154:76-82.

21. Pinto TJ, Farias DR, Rebelo F, Lepsch J, Vaz JS, Moreira JD, et al. Lower interpartum interval and unhealthy life-style factors are inversely associated with n-3 essential fatty acids changes during pregnancy: a prospective cohort with Brazilian women. PLoS One. 2015;10(3):e0121151. 
22. Verly-Miguel MV, Farias DR, Pinto Tde J, Lepsch J, Nardi AE, Kac G. Serum docosahexaenoic acid (DHA) is inversely associated with anxiety disorders in early pregnancy. J Anxiety Disord. 2015;30:34-40.

23. Makrides M, Gibson RA, McPhee AJ, Yelland L, Quinlivan J, Ryan P. Effect of DHA supplementation during pregnancy on maternal depression and neurodevelopment of young children: a randomized controlled trial. JAMA. 2010;304(15):1675-83.

24. Freeman MP, Davis M, Sinha P, Wisner KL, Hibbeln JR, Gelenberg AJ. Omega-3 fatty acids and supportive psychotherapy for perinatal depression: a randomized placebo-controlled study. J Affect Disord. 2008;110(1-2):142-8.

25. Su KP, Huang SY, Chiu TH, Huang KC, Huang CL, Chang HC, et al. Omega-3 fatty acids for major depressive disorder during pregnancy: results from a randomized, double-blind, placebo-controlled trial. J Clin Psychiatry. 2008; 69(4):644-51.

26. Appleton KM, Sallis HM, Perry R, Ness AR. Churchill R: omega-3 fatty acids for major depressive disorder in adults: an abridged Cochrane review. BMJ Open. 2016;6(3):e010172.

27. Appleton KM, Rogers PJ, Ness AR. Updated systematic review and metaanalysis of the effects of n-3 long-chain polyunsaturated fatty acids on depressed mood. Am J Clin Nutr. 2010;91(3):757-70.

28. Bloch MH, Hannestad J. Omega-3 fatty acids for the treatment of depression: systematic review and meta-analysis. Mol Psychiatry. 2012; 17(12):1272-82.

29. Miller BJ, Murray L, Beckmann MM, Kent T, Macfarlane B. Dietary supplements for preventing postnatal depression. Cochrane Database Syst Rev. 2013;10:CD009104

30. Appleton KM, Sallis HM, Perry R, Ness AR, Churchill R. Omega-3 fatty acids for depression in adults. Cochrane Database Syst Rev. 2015;11:CD004692.

31. Lewin GA, Schachter HM, Yuen D, Merchant P, Mamaladze V, Tsertsvadze A. Effects of omega-3 fatty acids on child and maternal health. Evid Rep Technol Assess (Summ). 2005;118:1-11.

32. Sheehan DV, Lecrubier $Y$, Sheehan $\mathrm{KH}$, Amorim $\mathrm{P}$, Janavs J, Weiller $\mathrm{E}$, et al. The Mini-international neuropsychiatric interview (M.I.N.I.): the development and validation of a structured diagnostic psychiatric interview for DSM-IV and ICD-10. J Clin Psychiatry. 1998;59(Suppl 20):22-33. quiz 4-57.

33. Teofilo MM, Farias DR, Pinto Tde J, Vilela AA, Vaz Jdos S, Nardi AE, et al. $\mathrm{HDL}$-cholesterol concentrations are inversely associated with Edinburgh postnatal depression scale scores during pregnancy: results from a Brazilian cohort study. J Psychiatr Res. 2014;58:181-8.

34. Murray D, Cox JL. Screening for depression during pregnancy with the edinburgh depression scale (EDDS). J Reprod Infant Psychol. 1990;8(2):99-107.

35. Doornbos B, van Goor SA, Dijck-Brouwer DA, Schaafsma A, Korf J, Muskiet FA. Supplementation of a low dose of DHA or DHA+AA does not prevent peripartum depressive symptoms in a small population based sample. Prog Neuro-Psychopharmacol Biol Psychiatry. 2009;33(1):49-52

36. Santos IS, Matijasevich A, Tavares BF, Barros AJ, Botelho IP, Lapolli C, et al. Validation of the Edinburgh postnatal depression scale (EPDS) in a sample of mothers from the 2004 Pelotas birth cohort study. Cad Saude Publica. 2007:23(11):2577-88.

37. OMd S. Classificação de Transtornos Mentais e de Comportamento da CID-10. Critérios diagnósticos para pesquisa. Editora Artes Médicas: Porto Alegre; 1998.

38. APA. American Psychiatric Association. Diagnostic and statistical Manual of mental disorders DSM-IV-TR fourth edition (text revision). USA: American Psychiatric Publishing; 2000.

39. Arab L, Akbar J. Biomarkers and the measurement of fatty acids. Public Health Nutr. 2002;5(6A):865-71.

40. Lin YH, Salem N Jr, Wells EM, Zhou W, Loewke JD, Brown JA, et al. Automated high-throughput fatty acid analysis of umbilical cord serum and application to an epidemiological study. Lipids. 2012;47(5):527-39.

41. Masood MA, Salem N Jr. High-throughput analysis of plasma fatty acid methyl esters employing robotic transesterification and fast gas chromatography. Lipids. 2008;43(2):171-80.

42. Lohman TG, Roche AF, Martorell R. Anthropometric Standarization reference Manual. Champaign, IL: Human Kinetics Books; 1988.

43. Sichieri R, Everhart MD. Validity of a Brazilian food frequency questionnaire against dietary recalls and estimated energy intake. Nutr Res. 1998;18:1649-59.

44. Lopes TS, Ferrioli E, Hoffman D, Sichieri R, Pereira RA. Validation of estimates of energy intake by food frequency questionnaire against doubly labeled water. Rev Chil Nutr. 2009;36:614.

45. Lepsch J, Vaz JS, Moreira JD, Pinto TJ, Soares-Mota M, Kac G. Food frequency questionnaire as an indicator of the serum composition of essential n-3 and n-6 polyunsaturated fatty acids in early pregnancy, according to body mass index. J Hum Nutr Diet. 2015;28(1):85-94.

46. Figueira P, Correa H, Malloy-Diniz L, Romano-Silva MA. Edinburgh postnatal depression scale for screening in the public health system. Rev Saude Publica. 2009;43(Suppl 1):79-84.

47. Llorente AM, Jensen CL, Voigt RG, Fraley JK, Berretta MC, Heird WC. Effect of maternal docosahexaenoic acid supplementation on postpartum depression and information processing. Am J Obstet Gynecol. 2003;188(5):1348-53.

48. Mozurkewich EL, Clinton CM, Chilimigras JL, Hamilton SE, Allbaugh LJ, Berman DR, et al. The mothers, omega-3, and mental health study: a double-blind, randomized controlled trial. Am J Obstet Gynecol. 2013; 208(4):313 e1-9.

49. Martins JG. EPA but not DHA appears to be responsible for the efficacy of omega-3 long chain polyunsaturated fatty acid supplementation in depression: evidence from a meta-analysis of randomized controlled trials. J Am Coll Nutr. 2009;28(5):525-42.

50. Walsh BT, Seidman SN, Sysko R, Gould M. Placebo response in studies of major depression: variable, substantial, and growing. JAMA. 2002; 287(14):1840-7.

51. Figueiredo $B$, Conde A. Anxiety and depression in women and men from early pregnancy to 3-months postpartum. Arch Womens Ment Health. 2011; 14(3):247-55.

52. Pinto TJ, Vilela AA, Farias DR, Lepsch J, Cunha GM, Vaz JS, Factor-Litvak P, Kac G: Serum n-3 polyunsaturated fatty acids are inversely associated with longitudinal changes in depressive symptoms during pregnancy. Epidemiol Psychiatr Sci. 2017;26(2):157-68. doi:10.1017/S204579601500116X.

53. Vilela AA, Farias DR, Eshriqui I, Vaz Jdos S, Franco-Sena AB, Castro MB, et al. Prepregnancy healthy dietary pattern is inversely associated with depressive symptoms among pregnant Brazilian women. J Nutr. 2014;144(10):1612-8.

54. Guardino CM, Schetter CD. Coping during pregnancy: a systematic review and recommendations. Health Psychol Rev. 2014;8(1):70-94.

55. Figueiredo B. Conde A. Anxiety and depression symptoms in women and men from early pregnancy to 3-months postpartum: parity differences and effects. J Affect Disord. 2011:132(1-2):146-57.

56. Kris-Etherton PM, Innis S, Ammerican Dietetic A, Dietitians of C. Position of the American Dietetic association and dietitians of Canada: dietary fatty acids. J Am Diet Assoc. 2007;107(9):1599-611.

57. IOM. Analysis of the balancing of benefits and risks of seafood consumption. In: Nesheim MC, Yaktine AL, editors. Seafood choices: balancing benefits and risks. Washington, DC: National Academic Press; 2007. p. 195-216.

58. Koletzko B, Cetin I, Brenna JT. Dietary fat intakes for pregnant and lactating women. Br J Nutr. 2007;98(5):873-7.

59. Hallahan B, Ryan T, Hibbeln JR, Murray IT, Glynn S, Ramsden CE, et al. Efficacy of omega-3 highly unsaturated fatty acids in the treatment of depression. Br J Psychiatry. 2016;209(3):192-201.

\section{Submit your next manuscript to BioMed Central and we will help you at every step:}

- We accept pre-submission inquiries

- Our selector tool helps you to find the most relevant journal

- We provide round the clock customer support

- Convenient online submission

- Thorough peer review

- Inclusion in PubMed and all major indexing services

- Maximum visibility for your research

Submit your manuscript at www.biomedcentral.com/submit
) Biomed Central 